\title{
Fractura luxación bipolar de clavícula: clavícula flotante
}

\author{
Luis Gerardo Domínguez Gasca, ${ }^{1}$ Francisco Javier Martínez Rodríguez, ${ }^{1}$ \\ Jorge Magaña Reyes, ${ }^{2}$ Luis Gerardo Domínguez Carrillo ${ }^{3}$
}

\section{Resumen}

Antecedentes: La fractura luxación bipolar de clavícula es una lesión extraordinaria, existen menos de 50 casos reportados en la literatura, ocurre a consecuencia de trauma directo de alta energía. Fue descrita inicialmente por Porral en 1831; por ser una lesión muy rara no existe consenso en cuanto al tratamiento. Caso clínico: Masculino de 50 años que sufrió traumatismo directo al caer de tres metros de altura sobre hombro izquierdo, fue atendido por empírico e inmovilizado por Velpeau, acudió a urgencias dos semanas después por presentar fiebre y supuración en región de articulación esternoclavicular. A la exploración: temperatura de $38^{\circ} \mathrm{C}$; zona de $2 \mathrm{~cm}$ de diámetro de pérdida de sustancia que permitía ver hueso sobre área de articulación esternoclavicular izquierda con supuración abundante. Los estudios mostraron fractura luxación bipolar de clavícula expuesta en su porción medial con infección activa.

Palabras clave: Fractura luxación bipolar de clavícula, clavícula flotante.

\section{Summary}

Background: Bipolar fracture dislocations of the clavicle are rare injuries, there are less of 50 cases reported. Usually it is result of high-energy direct trauma. Porral in 1831 described the first case. Management of these injuries has remained controversial ranging from non operative to aggressive surgery. Clinical case: A 50 years old male, who suffered three meters fall over left shoulder, attended initially by empiric with Velpeau for three weeks, patient comes to Emergency Room with fever and purulent material of left sternoclavicular joint exploration. Physical exploration showed: temperature of $38^{\circ} \mathrm{C} ; 2 \mathrm{~cm}$ in diameter area with loss of substance, including viewing area bone on left sternoclavicular joint with abundant purulent secretion. Studies showed bipolar dislocation fracture collarbone, exposed in its medial portion with active infection.

Key words: Bipolar fracture dislocations of the clavicle, floating clavicle.

\section{INTRODUCCIÓN}

Tanto las fracturas aisladas de clavícula como la luxación acromioclavicular son lesiones muy comunes, mientras que la fractura luxación bipolar de clavícula es extraordinariamente rara. Para que ocurra este tipo de lesión se requiere un trauma de alta energía. En la literatura anglosajona existen menos de 50 casos reportados, el primero por Porral ${ }^{1}$ en 1831, la serie más grande de 16 casos por Beckman ${ }^{2}$ en 1924, la de Sanders ${ }^{3}$ con seis casos en 1990 y por último, tres casos tratados por Dieme ${ }^{4}$ y su grupo en 2007, siendo el resto casos aislados, sumando desde su descripción 47 casos registrados (incluyendo el presente) en 182 años (1831-2016). Debido a su escasa frecuencia, el tratamiento es controvertido, ya que un tercio de los casos reportados ha sido manejado de manera conservadora y el resto ha recibido tratamiento quirúrgico. La revisión de los casos documentados indica que en el paciente joven se prefiere la opción quirúrgica, a 
diferencia del paciente de mayor edad, en el que prevalece la opinión de manejo conservador. A raíz de un paciente cuyo diagnóstico fue clavícula flotante con características especiales, efectuamos esta comunicación.

\section{CASO CLÍNICO}

Masculino de 50 años de edad que al trasladarse en bicicleta sufrió traumatismo directo al caer de tres metros de altura sobre hombro izquierdo, manifestando de inmediato dolor intenso en cintura escapular izquierda, deformidad de la región e imposibilidad de movimiento por dolor de la extremidad ipsilateral. Relata que acudió con empírico quien le "acomodó" y aplicó vendaje tipo Velpeau "muy apretado", dos semanas después de la lesión acude a urgencias por presentar fiebre y supuración en región esternoclavicular izquierda. A la exploración: temperatura de $38{ }^{\circ} \mathrm{C}$; TA 120/80; FC 100X'; FR 18X', se observan múltiples tatuajes, se retira vendaje serosanguinolento de olor fétido, encontrando zona de $2 \mathrm{~cm}$ de diámetro de pérdida de sustancia que permite ver hueso sobre área de articulación esternoclavicular izquierda (Figura 1), además de deformación de cintura escapular izquierda, el resto de la exploración de tórax y abdomen normal. El examen clínico muscular mostró: trapecio izquierdo y deltoides con calificación $3 / 5$ por dolor, resto de músculos examinados en extremidad superior izquierda en $4 / 5$ por desuso al compararlos con la extremidad contralateral, sensibilidad conservada, reflejos osteotendinosos normales al igual que pulsos arteriales y llenado capilar digital. Se tomó cultivo de zona de lesión en el momento de la debridación, se solicitó laboratorio, tomografía computarizada de tórax (Figuras 2 y 3), electrocardiograma y se le hospitalizó. Se inició tratamiento antibiótico empírico con ceftriaxona $1 \mathrm{~g}$

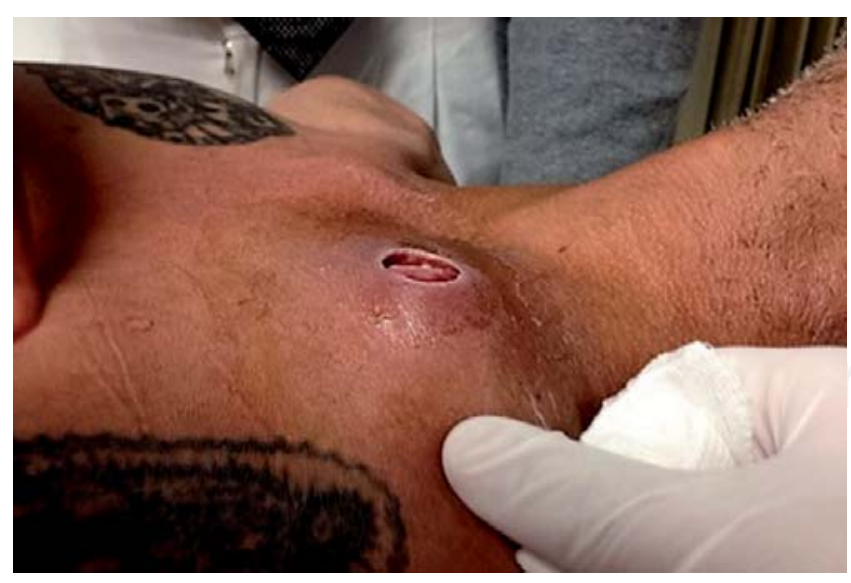

Figura 1. Pérdida de sustancia de $2 \mathrm{~cm}$ de diámetro sobre articulación esternoclavicular con fractura luxación en masculino de 50 años de edad. una vez al día y clindamicina 600 mg dos veces al día. El electrocardiograma y la telerradiografía de tórax con silueta cardiaca y campos pulmonares revelaron fractura luxación bipolar de clavícula izquierda, la tomografía con reconstrucción helicoidal confirmó sus características. La lesión proximal de clavícula se catalogó en AO 15.B de tipo III B según la clasificación de Gustillo-Andersen y la lesión distal de clavícula en AO 15.3A1. Al cabo de 48 horas el cultivo reportó Staphylococcus epidermidis. Se decidió después de 10 días de antibioticoterapia y con resultados de segundo cultivo negativo efectuar ostectomía de 1.4 cm de la porción medial de clavícula, reconstrucción del ligamento costoclavicular y cierre de área con colgajo con inmovilización mediante cabestrillo reforzado con cinturón en velcro por cuatro semanas. En el seguimiento entre 10 y 20 días el paciente mostró buena evolución sin dato de infección, a las cuatro semanas se retiraron puntos y se canalizó a rehabilitación, a los 60 días del programa de rehabilitación el paciente fue dado de alta con calificación 15/100 en la escala de DASH (Disability of the Arm Shoul-

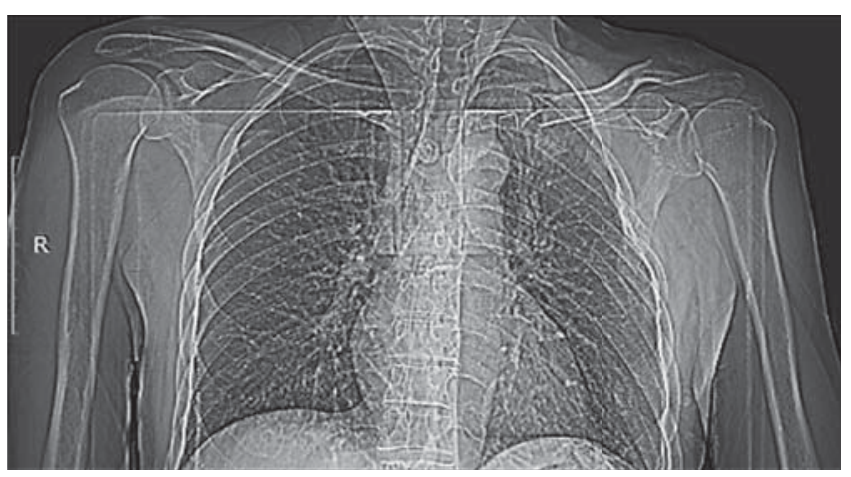

Figura 2. Pantograma de tórax (tomografía computarizada) que muestra luxación de articulación acromioclavicular izquierda y fractura distal de clavícula (AO 15.3A1), así como luxación esternoclavicular y fractura proximal de la clavícula izquierda (AO 15.B).

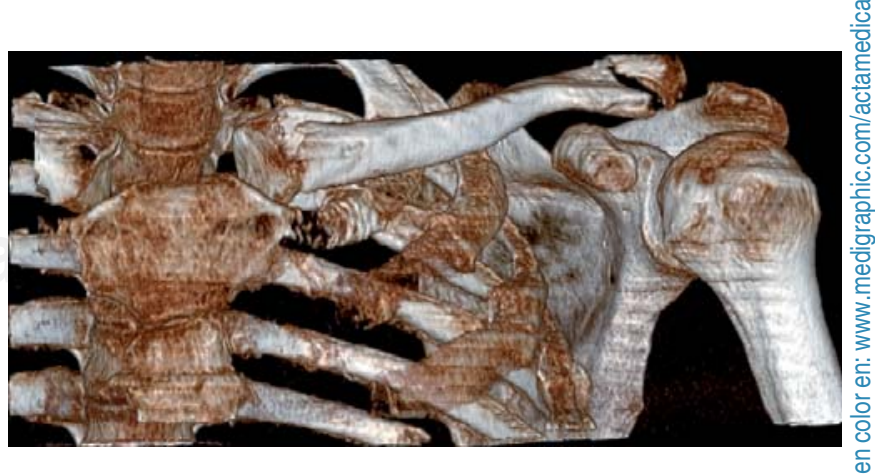

Figura 3. Tomografía computarizada con reconstrucción ® helicoidal que muestra: fractura luxación bipolar de clavícula $\frac{0}{.0}$ izquierda (clavícula flotante). 
der and Hand), con arcos de movilidad limitados en los últimos $10^{\circ}$ para la rotación interna y $15^{\circ}$ en abducción de hombro, sin dolor.

\section{DISCUSIÓN}

El término de fractura dislocación bipolar de la clavícula indica que existe fractura simultánea de la porción lateral y medial de la clavícula (fractura segmentaria), incluye luxación de las articulaciones acromioclavicular y esternoclavicular (EC), por lo que también se ha denominado clavícula flotante. Dada la rareza de la lesión se desconoce su incidencia y prevalencia. Esta lesión fue descrita inicialmente por Porral en 1831. De acuerdo con la revisión de Gouse ${ }^{5}$ et al. se infiere que prevalece en personas jóvenes en dos tercios de los casos y el resto en pacientes mayores de 50 años. En un periodo de 89 años (de 1924 a 2013) existen 24 casos reportados en la literatura, (33\%) se manejaron de manera conservadora, mientras que $(66 \%)$ recibieron tratamiento quirúrgico. Del total de casos reportados, 25\% $(\mathrm{n}=6)$ forman la serie que pertenece a Sanders. ${ }^{3}$

Desde el punto de vista de la etiopatogenia, el mecanismo teórico de esta compleja lesión segmentaria depende de un trauma de alta energía ${ }^{6}$ que requiere que la clavícula sea expuesta de manera secuencial a dos fuerzas, un golpe directo sobre la parte lateral del hombro, seguida de torsión del tronco. Inicialmente la clavícula actúa como brazo de palanca con punto de apoyo en los ligamentos conoide y trapezoide, lo que llega a producir subluxación o luxación (ya sea anterosuperior o anteroinferior) de la articulación esternoclavicular (EC) que puede ocasionar fractura proximal de la clavícula. Posteriormente existe subluxación o luxación de la articulación acromioclavicular y/o fractura de la porción distal de la clavícula. Debe tomarse en cuenta que el fulcro de estas lesiones son los ligamentos conoide y trapezoide, los cuales inicialmente están intactos, pero pueden llegar a romperse. La luxación EC por sí sola no es frecuente, alcanza 3\% de todas las luxaciones, siendo la luxación anterior de dos a tres veces más frecuente que la posterior y menos peligrosa.

En el caso que nos ocupa, el paciente además de sufrir esta rara lesión acudió a consulta tardíamente, dos semanas posteriores al traumatismo, con pérdida de sustancia de $2 \mathrm{~cm}$ de diámetro acompañada de supuración localizada sobre articulación EC, la cual era visible. Con respecto al mecanismo de lesión cutánea las posibilidades consideradas fueron: a) vendaje compresivo intenso sobre la fractura luxación EC que originó una lesión isquémica (úlcera de presión) que provocó infección al romperse la piel; b) lesión puntiforme que perforó la piel por fragmento óseo, que al tratar de reducirla, introdujo bacterias dentro de la zona fracturaria causando absceso que a su vez abrió hacia la piel y c) lesión directa en la articulación EC con machacamiento y escoriaciones del tejido de la zona que al no manejarse adecuadamente se infectó. Respecto al diagnóstico, en las radiografías simples la fractura luxación proximal de la clavícula puede pasar desapercibida, por lo que la tomografía computarizada ${ }^{7}$ es la herramienta diagnóstica de elección tanto para valorar la lesión como las patologías coexistentes. Es de vital importancia descartar lesiones asociadas, principalmente aquéllas producto de colisión de vehículos en movimiento como: compresión y laceración de la arteria subclavia, compresión mediastinal, neumotórax, ruptura esofágica, anormalidades de conducción cardiaca, lesión del plexo braquial, ruptura traqueal y síndrome de salida torácica. Al respecto el estudio de las lesiones asociadas a las fracturas de clavícula descritas en el estudio de Taitsman ${ }^{8}$ y su grupo muestra: $75 \%$ lesión pulmonar, incluyendo neumotórax unilateral o bilateral, contusión pulmonar y derrame; 35\% de los casos presentan fractura cervical o torácica; $40 \%$ fractura concomitante de escápula; 30\% lesión ipsilateral del miembro superior y trauma facial en $55 \%$ de los casos. En relación con las fracturas abiertas de clavícula, éstas son raras y se asocian primordialmente a lesión pulmonar y craneal, así como a afectación de la extremidad superior ipsilateral, siendo las lesiones vasculares infrecuentes.

En virtud de que las lesiones de la articulación EC son infrecuentes, ${ }^{9}$ la anatomía de la región no es familiar para la mayoría de los cirujanos ortopedistas y existe alto riesgo de complicaciones catastróficas tanto en el transoperatorio como en el postoperatorio, esencialmente en la luxación EC posterior. El consenso de manejo es que la inestabilidad de la articulación EC debe ser tratada inicialmente de manera conservadora; debe informarse al paciente que existe un alto riesgo de inestabilidad persistente con manejo no quirúrgico, la cual en general es bien tolerada y tiene leve impacto funcional en la mayoría de las personas. Los pacientes con luxación EC posterior requieren un manejo muy cuidadoso por la proximidad de los grandes vasos. La reducción temprana habitualmente es estable, debe efectuarse la estabilización quirúrgica si la reducción no es exitosa o persiste la inestabilidad, el éxito de la cirugía depende de la reparación del ligamento romboideo como de la cápsula articular.

Se han propuesto varias opciones quirúrgicas ${ }^{10}$ para la luxación anterior aguda y crónica de la articulación EC:

1. Sutura de los ligamentos esternoclavicular e interclavicular con reducción abierta suturando cápsula y ligamentos y fijación con alambre 17-37, utilizando la fijación de la sutura al esternón. 
2. Reconstrucción del ligamento esternoclavicular anterior. Se han utilizado diferentes injertos desde fascia lata o semitendinosos colocados en ocho, así como la porción medial del esternocleidomastoideo.

3. Una tercera opción es la reconstrucción del ligamento interclavicular con fascia lata o músculo subclavio, fijándolos a la segunda costilla, así como la tenodesis de la cabeza esternal del esternocleidomastoideo.

4. La resección de la clavícula proximal. Esta última opción no es recomendada por muchos autores a causa de sus malos resultados; sin embargo, para Groh ${ }^{11}$ y su grupo la resección medial de la clavícula es un tratamiento efectivo siempre y cuando el ligamento costoclavicular esté intacto o sea reconstruido durante la cirugía.

Con relación a la resección de la clavícula proximal, el estudio en cadáveres de Bisson ${ }^{12}$ aconseja resecciones no mayores de $1.2 \pm 0.3 \mathrm{~cm}$, insistiendo en la preservación o reconstrucción del ligamento costoclavicular (romboideo). Para la cirugía el borde rugoso clavicular del músculo pectoral sirve como referencia ósea para los tejidos blandos insertados alrededor de la articulación EC, el ligamento costoclavicular es el más grande e importante de la articulación, el cartílago articular cubre $67 \%$ de la superficie medial articular de la clavícula y su localización es anteroinferior, el músculo esternohioideo se inserta directamente sobre la parte posterior de la articulación y la porción proximal de la clavícula, el músculo esternotiroideo se inserta $9.5 \mathrm{~mm}$ inferior al margen posterosuperior del manubrio esternal y cursa $19.8 \mathrm{~mm}$ lateralmente a lo largo de la primera costilla, por lo que un plano avascular que sirve como zona segura para la disección es posterior a la articulación EC y anterior a los músculos esternohioideo y esternotiroideo.

Posterior a la cirugía se recomienda vendaje en ocho o en Velpeau de tres a seis semanas; el beneficio de éste no ha sido documentado en la literatura, los pacientes deben evitar elevar el brazo más de $60^{\circ}$, posterior a ello el incremento gradual de arcos de movimiento debe efectuarse con guía de rehabilitación. Los resultados funcionales ${ }^{13}$ a largo plazo (promedio de seguimiento de 9 años) en las escalas ASES y DASH de valoración de funcionalidad del miembro superior no muestran diferencia entre la reconstrucción de la articulación EC o la resección proximal de la clavícula, lográndose en ambos casos estabilidad siempre y cuando el ligamento costoclavicular sea preservado o reconstruido.

\section{CONCLUSIONES}

El consenso de manejo de clavícula flotante es que debe tratarse inicialmente de manera conservadora; existe alto riesgo de inestabilidad persistente con manejo no quirúrgico; la inestabilidad en general es bien tolerada y tiene leve impacto funcional en la mayoría de las personas. La estabilización quirúrgica puede efectuarse si la reducción no es exitosa o persiste la inestabilidad, el éxito de la cirugía depende de la reparación del ligamento romboideo como de la cápsula articular.

En caso de fractura abierta el manejo con antibióticos es imperativo, evitando colocar material de osteosíntesis y en caso de resección ósea de la porción medial de la clavícula se recomienda hacer resecciones no mayores de $1.5 \mathrm{~cm}$, insistiendo en la preservación o reconstrucción del ligamento costoclavicular.

\section{REFERENCIAS}

1. Porral D. Observation d'une double luxation de la clavicule droite. J Univ Hebd Med Chir. 1831; 2: 78-82.

2. Beckman T. A case of simultaneous luxation of both ends of the clavicle. Acta Chir Scandinavica. 1924; 56: 156-163.

3. Sanders JO, Lyons FA, Rockwood CA Jr. Management of dislocations of both ends of the clavicle. J Bone Joint Surg Am. 1990; 72 (3): 399402.

4. Dieme C, Bousso A, Sane A, Sané JC, Niane M, Ndiaye A et al. Bipolar dislocation of the clavicle or floating clavicle. A report of 3 cases. Chir Main. 2007; 26 (2): 113-116.

5. Gouse M, Jacob KM, Poonnoose PM. Traumatic floating clavicle: a case report and literature review. Case Rep Orthop. 2013; 2013: 386089. doi: 10.1155/2013/386089.

6. Yurdakul E, Salt Ö, Uzun E, Doğar F, Güney A, Durukan P. Traumatic floating clavicle. Am J Emerg Med. 2012; 30 (9): 2097.e3-2097.e5.

7. Mirka H, Ferda J, Baxa J. Multidetector computed tomography of chest trauma: indications, technique and interpretation. Insights Imaging. 2012; 3 (5): 433-449.

8. Taitsman LA, Nork SE, Coles CP, Barei DP, Agel J. Open clavicle fractures and associated injuries. J Orthop Trauma. 2006; 20 (6): 396-399.

9. Thut D, Hergan D, Dukas A, Day M, Sherman OH. Sternoclavicular joint reconstruction--a systematic review. Bull NYU Hosp Jt Dis. 2011; 69 (2): 128-135.

10. Lee JT, Campbell KJ, Michalski MP, Wilson KJ, Spiegl UJ, Wijdicks CA et al. Surgical anatomy of the sternoclavicular joint: a qualitative and quantitative anatomical study. J Bone Joint Surg Am. 2014; 96 (19): e166.

11. Groh GI, Wirth MA. Management of traumatic sternoclavicular joint injuries. J Am Acad Orthop Surg. 2011; 19 (1): 1-7.

12. Bisson LJ, Dauphin N, Marzo JM. A safe zone for resection of the medial end of the clavicle. J Shoulder Elbow Surg. 2003; 12 (6): 592-594.

13. Panzica M, Zeichen J, Hankemeier S, Gaulke R, Krettek C, Jagodzinski $M$. Long-term outcome after joint reconstruction or medial resection arthroplasty for anterior SCJ instability. Arch Orthop Trauma Surg. 2010; 130 (5): 657-665. 